\title{
Series-Parallel Power System Optimization Using Firefly Algorithm
}

\author{
Khaled Guerraiche ${ }^{1}$, Mostefa Rahli ${ }^{1}$, Abdelkader Zeblah ${ }^{2}$ and Latifa Dekhici ${ }^{1}$ \\ ${ }^{1}$ University of Sciences and the Technology (USTO-MB), 31000 Oran, Algeria \\ ${ }^{2}$ University of Sidi Bel Abbes, B.P. 22000, Sidi Jillali, Sidi Bel Abbes, Algeria. \\ khguerraiche@yahoo.fr
}

Abstract: In this paper, we try to solve the Redundancy Optimization Problem (ROP) using a recent bio-inspired meta-heuristic that is Firefly Algorithm (FA) combined to Universal Moment Generating Function (UMGF).The problem consists on selecting the appropriate components from a multi-state system (MSS) given the minimum cost. The solution must also fulfill a system reliability constraint. The reliability in our work is considered as the ability to satisfy the demand and is calculated using u-tranform technique. Our algorithm was tested on a system contained multiple choices of five subsystems from a list of product available in the market. The components of each subsystem are characterized by their cost, performance (capacity) and availability. The results obtained by the proposed algorithm are compared with several algorithm founds in literature.

Keywords: Firefly algorithm, Multi-state system (MSS), reliability, Power system design, Universal moment generating function (UMGF)

\section{Introduction}

The role of systems analysts and designers becomes very challenging due to the diversity of components technology and versions. Especially, we cannot guarantee high reliability and the required customer demand without ensuring sometimes a high cost. That why, only the use of computer tools and efficient meta-heuristics can solve such optimization problem. Actually, reliability optimization lets us assume that both of components and series-parallel system may be in other states than the two habitual states that are complete working and total failure states. They can still operational in other levels of performance. Thus, the approaching optimization methods seem to be preferable than exact classic methods since they can reach solution closer to required reliability. Recently much works treat cost and reliability optimization of seriesparallel systems using approaching methods. The problem of total cost minimization, subject to availability constraints, is well known as the redundancy optimization problem (ROP). The ROP is studied in many different forms as summarized in [1,2]. The ROP for the multi-state reliability was introduced in $[3,4,5]$. Many meta-heuristic were used such as: Genetic Algorithms [5], Particles Swarm Optimization and Local Search [6], Harmony Search [7], Ant Colony Optimization [8], Tabu Search [9] and Immune Algorithm [10].

This work proposes a Firefly meta-heuristic algorithm to solve the redundancy optimization problem. The algorithm has to find the optimal multi-state structure (MSS) design that may be a power station coal transportation system which supplies the boiler consists of five subsystems. The optimal solution corresponds to the minimum cost and is subject to the requirement of meeting the demand with the desirable level of reliability [11]. To evaluate the reliability for series-parallel system structure, we develop a procedure which is based on universal moment generating function (UMGF) [3]. An approach to model the redundancy optimization problem is suggested in [4-12]. In this approach costs of components are defined as explicit analytical function of component capacities and the same reliability index value is assigned to all components of a given type, regardless of their capacity. In [13, 14], respectively a genetic algorithm and ant colony approaches are used as an optimization technique to solve the problem.

The remainder of this paper is organized as follows: the second Section of the paper consists on a general description of model used and redundancy optimization problem

Received: October $12^{\text {th }}, 2014$. Accepted: March $7^{\text {th }}, 2015$

DOI: $10.15676 /$ ijeei.2015.7.1.7 
formulation. In the third section, we describe the reliability estimation method using the UMGF technique. The fourth Section describes the basic Firefly algorithm and its adaptation to the problem. In last section, a comparative study between algorithm proposed and other published algorithms in literature

\section{Formulation of Redundancy Optimization Problem (ROP)}

Let us consider a power system containing $\mathrm{N}$ electrical power components connected in series- parallel as sketched in Figure 1. There has been much interest in power production models, where new redesign is considered. To formulate the new redesign problem, let consider a series-parallel power system containing $n$ subsystems $\mathrm{i}=1,2 \ldots \ldots, \mathrm{N}$ in series. Each component turn contains a number of different components connected in parallel. All components of any given subsystem belong to different version v. Components a characterized by their Reliabilitv $\left(\mathrm{A}_{\mathrm{ii}}\right)$, cost $\left(\mathrm{C}_{\mathrm{ij}}\right)$ and performance $\left(\Xi_{:: \text {: }}\right)$ according to their version
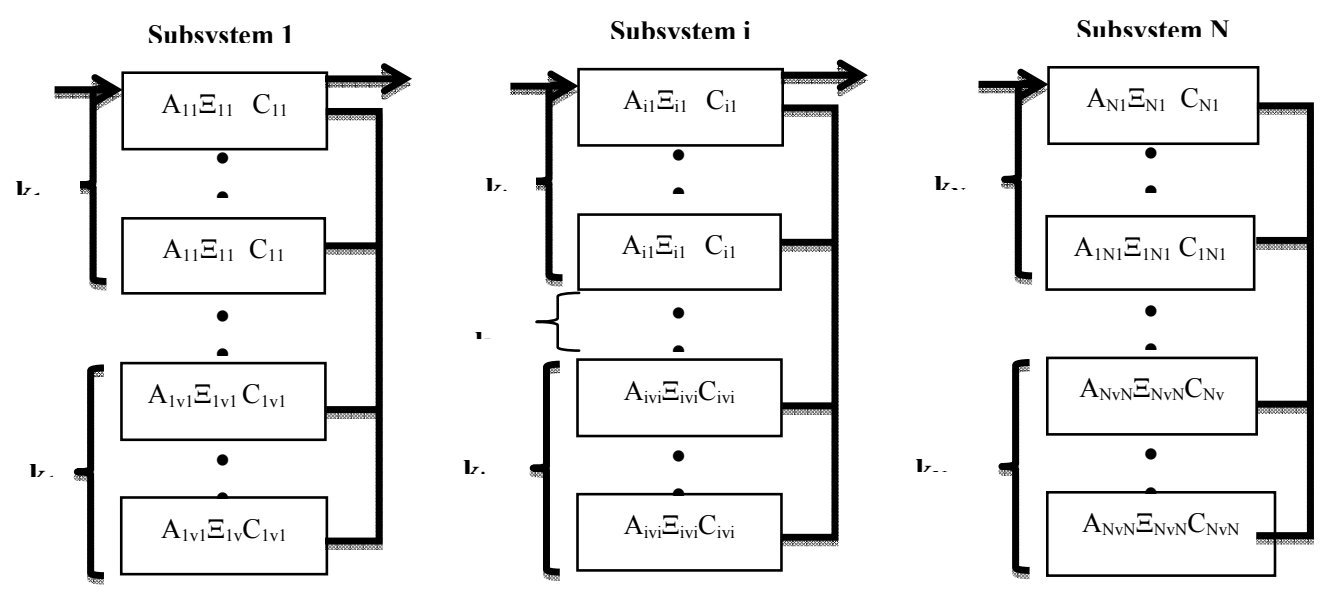

Figure 1. Series-Parallel System.

The structure of subsystem $\mathrm{i}$ can be defined by the numbers of parallel components $\mathrm{k}_{\mathrm{ij}}$ for $1 \leq j \leq V_{i}$, where $V_{i}$ is the number of versions available for component of type $i$. The entire system can therefore be defined by the set of triplets $\left\{\mathrm{A}_{\mathrm{ij}}, \Xi_{\mathrm{ij}}, \mathrm{C}_{\mathrm{ij}}\right\}\left(1 \leq \mathrm{i} \leq \mathrm{N}, 1 \leq \mathrm{j} \leq \mathrm{V}_{\mathrm{i}}\right) . \mathrm{K}$ represents the initial system structure. In fact, for given-K, the total cost of the power system structure can be calculated as

$$
C=\sum_{i=1}^{n} \sum_{j=1}^{V_{i}} k_{i j} c_{i j}
$$

Usually in electrical power energy, the Loss of Load Probability index (LOLP) and the Expected Energy Not Supplied (EENS) in operation period $T$ are used for reliability estimation [15]. This index measures the probability that the load demand will not be met. Generally the load demand is represented by discrete random curve. If the time period of load is the set of $M$ intervals, with duration $T_{l}(l=1, \ldots, M)$, and each demand level $W_{l}$ has $T_{l}$ duration, the LOLP is calculated as follows:

$$
\mathbf{L O L P}=\frac{1}{\sum_{l=1}^{M} T_{l}} \sum_{l=1}^{M} P\left(\Xi_{s} \succ W_{l}\right) T_{l}
$$


and

$$
\text { EENS }=\frac{1}{\sum_{l=1}^{M} T_{l}} \sum_{l=1}^{M} P\left(W_{l} \geq \Xi_{s}\right) T_{l}
$$

Where $\boldsymbol{P}\left(\Xi_{s} \leq \boldsymbol{W}_{\boldsymbol{l}}\right)$ represents the probability that the total system capacity $\Xi_{s}$ is lower than the demand level $w_{j}$ (EENS). All capacities production and demand are defined as a percentage of their total nominal value. The cumulative load curve is represented by vectors $W=\left\{W_{l}\right\}$ and $\boldsymbol{T}=\left\{\boldsymbol{T}_{\boldsymbol{l}}\right\}$ who is known for every power system.

The measure of reliability system is defined by $A$ index in reference [5], given by the expression $\boldsymbol{A}=1-\operatorname{LOLP}[16,17,18]$. This index will be compared and must be not less than some preliminarily specified level $A_{0}$

\section{A. Optimal Design Optimization}

The multi-state electrical power system redundancy optimization problem can be formulated as follows: find the minimal cost system configuration $\mathrm{k}_{1}, \mathrm{k}_{2, \ldots}, \mathrm{k}_{\mathrm{N}}$, such that the corresponding reliability exceeds or equal the specified reliability $A_{0}$. That is:

Minimize

$$
C=\sum_{i=1}^{n} \sum_{j=1}^{V_{i}} k_{i j} c_{i j}
$$

Subject To

$$
A\left(d, t, k_{1}, k_{2}, \ldots, k_{N}\right) \geq A_{0}
$$

\section{Reliability Estimation Method Based on UMGF}

The last few years have seen the appearance of a number of works presenting various methods of quantitative estimation of systems consisting of devices that have a range of working levels in $[19,20]$. Usually one considers reducible systems. In general, for the series connection, the level of working is determined by the worst state observed for any one of the devices, while for parallel connection the performance is obtained by adding the performances of the parallel devices. However, such the approach is not applicable for the majority of real systems. In this paper, the procedure used is based on the universal z-transform, which is a modern mathematical technique introduced by [21]. This method, convenient for numerical implementation, is proved to be very effective for high dimension combinatorial problems. In the literature, the universal z-transform is also called UMGF or simply u-transform.

The UMGF of a discrete random variable $\Xi$ is defined as a polynomial:

$$
\boldsymbol{u}(\mathbf{z})=\sum_{j=1}^{J} \boldsymbol{P}_{j} \mathbf{z}^{\Xi_{j}}
$$

Where the variable $\Xi$ has $J$ possible values and $P_{j}$ is the probability that $\Xi$ is equal to $\Xi \mathrm{j}$.

The probabilistic characteristics of the random variable $\Xi$ can be found using the function $\mathrm{u}(\mathrm{z})$. In particular, if the discrete random variable $\Xi$ is the MSS stationary output performance, the availability A is given by the probability $\operatorname{prob}(\Xi \geq \mathrm{W})$ which can be defined as follows: 


$$
\operatorname{Proba}(\Xi \geq \mathbf{W})=\Phi\left(u(z) z^{-W}\right)
$$

Where $\Phi$ is a distributive operator defined by expressions (7) and (8):

$$
\begin{aligned}
& \Phi\left(\boldsymbol{P z}^{\sigma-\mathbf{W}}\right)=\left\{\begin{array}{l}
\boldsymbol{P}, \text { if } \sigma \geq \mathbf{W} \\
0, \text { if } \sigma<\mathbf{W}
\end{array}\right. \\
& \Phi\left(\sum_{j=1}^{\boldsymbol{J}} \boldsymbol{P}_{j} \mathbf{z}^{\Xi_{j}-\mathbf{W}}\right)=\sum_{j=1}^{J} \Phi\left(\boldsymbol{P}_{j} \mathbf{z}^{\Xi_{j}-\mathbf{W}}\right)
\end{aligned}
$$

It can be easily shown that equations (7)-(8) meet condition Proba $(\Xi \geq \mathrm{W})=\sum_{\Xi_{\boldsymbol{j}} \geq \mathbf{W}} \boldsymbol{P}_{\boldsymbol{j}}$. By using the operator $\Phi$, the coefficients of polynomial $u(z)$ are summed for every term with $\Xi j \geq$ $\mathrm{W}$, and the probability that $\Xi$ is not less than some arbitrary value W is systematically obtained.

Consider single devices with total failures and each device $\mathrm{i}$ has nominal performance $\Xi_{\mathrm{i}}$ and reliability Ai. The UMGF of such an device has only two terms can be defined as:

$$
u_{i}(\mathbf{z})=\left(1-A_{i}\right) z^{0}+A_{i} z^{\Xi_{i}}=\left(1-A_{i}\right)+A_{i} z^{\Xi_{i}}
$$

To evaluate the MSS availability of a series-parallel system, two basic composition operators are introduced.

These operators determine the polynomial $\mathrm{u}(\mathrm{z})$ for a group of devices.

\section{A. Parallel devices}

Let consider a system device $\mathrm{n}$ containing Jn devices connected in parallel. The total performance of the parallel system is the sum of performances of all its devices. In power systems, the term capacity is usually used to indicate the quantitative performance measure of a device [12] Examples: generating capacity for a generator, carrying capacity for an electric transmission line, etc. Therefore, the total performance of the parallel unit is the sum of capacities (performances) [13]. The u-function of MSS device $\mathrm{n}$ containing $\mathrm{Jn}$ parallel devices can be calculated by using the $\mathfrak{I}$ operator:

$$
u_{p}(z)=\mathfrak{J}\left(u_{1}(z), u_{2}(z), \ldots, u_{n}(z)\right), \text { where } \mathfrak{T}\left(\Xi_{1}, \Xi_{2}, \ldots, \Xi_{n}\right)=\sum_{i=1}^{n} \Xi_{i}
$$

Therefore for a pair of devices connected in parallel:

$$
\begin{aligned}
& \mathfrak{J}\left(u_{1}(z), u_{2}(z)\right)=\mathfrak{I}\left(\sum_{i=1}^{n} P_{i} z^{a_{i}}, \sum_{j=1}^{m} Q_{j} z^{b_{j}}\right) \\
& =\sum_{i=1}^{n} \sum_{j=1}^{m} P_{i} Q_{j} z^{a_{i}+b_{j}}
\end{aligned}
$$

The parameters $a_{i}$ and $b_{j}$ are physically interpreted as the performances of the two devices. $\mathrm{n}$ and $\mathrm{m}$ are numbers of possible performance levels for these devices. $\mathrm{P}_{\mathrm{i}}$ and $\mathrm{Q}_{\mathrm{j}}$ are steadystate probabilities of possible performance levels for devices. One can see that the $\mathfrak{J}$ operator 
is simply a product of the individual $u$-functions. Thus, the device UMGF is: $\boldsymbol{u}_{\boldsymbol{p}}(\mathbf{z})=\prod_{\boldsymbol{j}=1}^{\boldsymbol{J}_{n}} \boldsymbol{u}_{\boldsymbol{j}}(\mathbf{z})$.

Given the individual UMGF of devices defined in equation (11), we have:

$u_{p}(\mathbf{z})=\prod_{j=1}^{J_{n}}\left(1-A_{j}+A_{j} z^{\Xi_{i}}\right)$.

B. Series devices

When the devices are connected in series, the device with the least performance becomes the bottleneck of the system. This device therefore defines the total system productivity. To calculate the u-function for system containing $\mathrm{m}$ devices connected in series, the operator $\delta$ should be used:

$$
\begin{aligned}
& \boldsymbol{u}_{s}(\mathbf{z})=\delta\left(\boldsymbol{u}_{1}(\mathbf{z}), \boldsymbol{u}_{2}(\mathbf{z}), \ldots, \boldsymbol{u}_{\boldsymbol{m}}(\mathbf{z})\right) \text {, where } \delta\left(\Xi_{1}, \Xi_{2}, \ldots, \Xi_{m}\right)=\min \left\{\Xi_{1}, \Xi_{2}, \ldots, \Xi_{m}\right\} \text { so that } \\
& \delta\left(u_{1}(z), u_{2}(z)\right)=\delta\left(\sum_{i=1}^{n} P_{i} z^{a_{i}}, \sum_{j=1}^{m} \boldsymbol{Q}_{j} z^{b_{j}}\right) \\
& \left.=\sum_{i=1}^{n} \sum_{j=1}^{m} P_{i} Q_{j} z^{\min \left\{a_{i},\right.} b_{j}\right\}
\end{aligned}
$$

Applying composition operators $\mathfrak{I}$ and $\delta$ consecutively, one can obtain the UMGF of the entire series-parallel system. To do this we must first determine the individual UMGF of each device.

\section{Devices With Total Failures}

Let consider the usual case where only total failures are considered and each subsystem of type $\mathrm{i}$ and version $\mathrm{v}_{\mathrm{i}}$ has nominal performance $\Xi$ iv and availability $\mathrm{A}_{\mathrm{ij}}$. In this case, we have: $\operatorname{Proba}\left(\Xi=\Xi_{i j}\right)=A_{i j}$ and $\operatorname{Proba}(\Xi=\mathbf{W})=1-A_{i j}$. The UMGF of such a device has only two terms can be defined as in equation (9) by $\boldsymbol{u}^{*}{ }_{i}(\mathbf{z})=\left(1-A_{i j}\right) \mathbf{z}^{0}+A_{i j} z^{\Xi_{i j}}=1-A_{i j}+A_{i j} z^{\Xi_{i j}}$. Using the $\mathfrak{I}$ operator, we can obtain the UMGF of the i-th system device containing $k_{i}$ parallel devices $u_{i}(z)=\left(u^{*}{ }_{i}(z)\right)^{k_{i}}=\left(A_{i j} z^{\Xi_{i j}}+\left(1-A_{i j}\right)\right)^{k_{i}}$.

The UMGF of the entire system containing n system devices connected in series composed with identical elements is:

$$
u_{s}(z)=\delta\left(\begin{array}{l}
\left(A_{1 j} z^{\Xi_{1 j}}+\left(1-A_{1 j}\right)\right)^{k_{1}}, \\
\left(A_{2 j} z^{\Xi_{2 j}}+\left(1-A_{2 j}\right)\right)^{k_{2}}, \ldots, \\
\left(A_{n j} z^{\Xi_{n j}}+\left(1-A_{n j}\right)\right)^{k_{n}}
\end{array}\right)
$$

To evaluate the probability $\operatorname{Pr} \operatorname{oba}(\Xi \geq W)$ for the entire system, the operator $\Phi$ is applied to equation (10): 


$$
\operatorname{Pr} \operatorname{oba}(\Xi \geq W)=\Phi\left(u_{s}(z) z^{-W}\right)
$$

\section{The Firefly Optimization Approach}

The problem formulated in this paper is a complicated combinatorial optimization problem. The total number of different solutions to be examined is very large, even for rather small problems. An exhaustive examination of the enormous number of possible solutions is not feasible given reasonable time limitations. Thus, because of the search space size of the ROP for MSS, a new algorithm is developed in this section. It consists in an adaptation of the Firefly meta-heuristic to our problem. Firefly algorithm (FA), a recently developed meta-heuristic optimization algorithm, is employed in this paper to solve the proposed optimization problems since the superiority of FA over some other meta-heuristic optimization algorithms was reported in $[22,23,24]$.

\section{A. Fireflies in Nature}

Fireflies, belong to family of Lampyridae, are small winged beetles capable of producing a cold light flashes in order to attract mates. They are believed to have a capacitor-like mechanism, that slowly charges until the certain threshold is reached, at which they release the energy in the form of light, after which the cycle repeats $[25,26]$. The basic principle and its implementation in our problems are briefly introduced in the following paragraphs.

\section{B. Basic Principles of Firefly Algorithm}

Firefly algorithm inspired by the flashing behavior of fireflies was recently put forth by Yang $[22,23,24]$. The fundamental functions of flashing light of fireflies are to communicate (like attracting mating partners) and to attract potential prey. Inspired by this nature, the firefly algorithm was developed by idealizing some of the flashing characteristics of fireflies and representing each individual solution of optimization problem as a firefly in population.

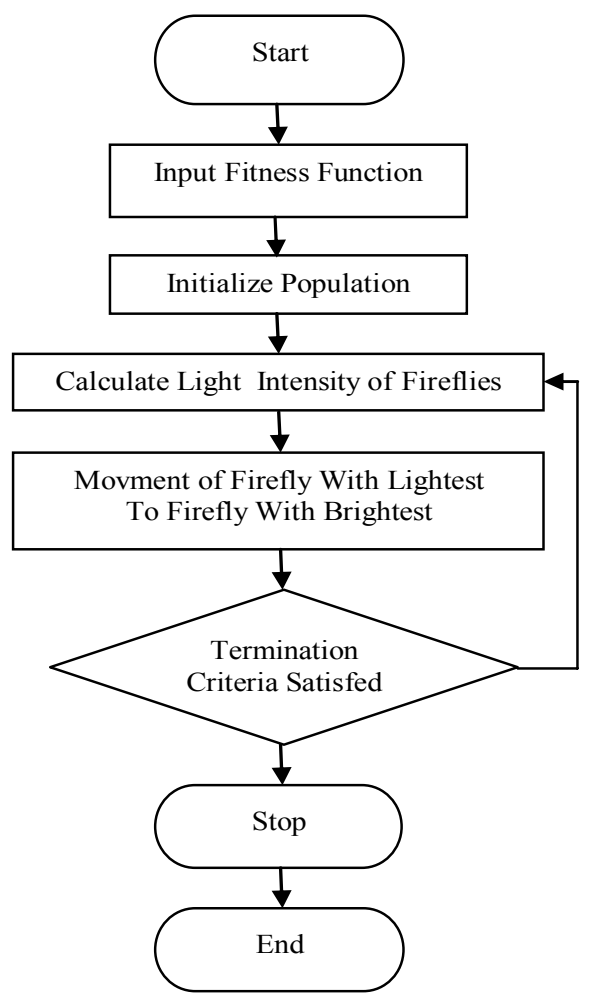

Figure 2 . The flow diagram of firefly algorithm 
Three major idealized rules are [22, 23][27, 28]:

- All fireflies in the population are unisex so that any individual firefly will be attracted at other fireflies;

- For any pair of fireflies, the less bright one will move towards the brighter one. The attractiveness of a firefly is proportionally related to the brightness which decreases with increasing distance between two fireflies;

- The brightness of a firefly is proportionally related to the value of objective function in the similar way to the fitness in genetic algorithm. The procedure of implementing the FA for a maximum optimization problem is summarized by the pseudo code shown in Figure 2 [22, $23,24,25][28]$.

C. Parameters

In the firefly algorithm, there are five important issues:

- Light Intensity: In the simplest case for minimum optimization problems, the brightness I of a firefly at a particular location $\mathrm{x}$ can be chosen as $\mathrm{I}(\mathrm{x}) \propto 1 / \mathrm{f}(\mathrm{x})$.

- Attractiveness: In the firefly algorithm, the main form of attractiveness function can be any monotonically decreasing functions such as the following generalized form:

$$
\boldsymbol{\beta}_{\mathrm{i}, \mathrm{j}}=\boldsymbol{\beta}_{0}^{*} e^{-\gamma r_{i, j}^{m}}
$$

Where $r$ is the distance between two fireflies, $\boldsymbol{\beta}_{0}^{*}$ is the attractiveness at $r=0$ and $\gamma$ is a fixed light absorption coefficient.

- Distance: The distance between any two fireflies $\mathrm{i}$ and $\mathrm{j}$ at $x_{i}$ and $x_{j}$ can be the Cartesian distance as follows:

$$
r_{i, j}=\sqrt{\sum_{k=1}^{d}\left(x_{i, k}-x_{j, k}\right)^{2}}
$$

Where $x_{i, k}$ is the $k^{\text {th }}$ component of the $i^{\text {th }}$ firefly.

- Movement: The movement of a firefly $i$ attracted to another more attractive (brighter) firefly $j$, is determined by

$$
x_{i}=\left(1-\beta_{i, j}\right) x_{i}+\beta_{i, j} x_{j}+\alpha(\operatorname{rand}-1 / 2)
$$

where the first and second term is due to the attraction while the third term is randomization with $\alpha$ being the randomization parameter and "rand" is a random number generator uniformly distributed in $[0,1]$.

\section{Experimental Results and Discussion}

A. Power Design Test Example

A power station coal transportation system which supplies the boiler consists of five basic components [5]:

1. Primary feeder which loads the coal from the bin to the primary conveyor.

2. Primary conveyor which transports the coal to the stacker-reclaimer.

3. Stacker-reclaimer which lifts the coal up to the burner level.

4. Secondary feeder which loads the secondary conveyor.

5. Secondary conveyor which supplies the burner feeding system of the boiler. 


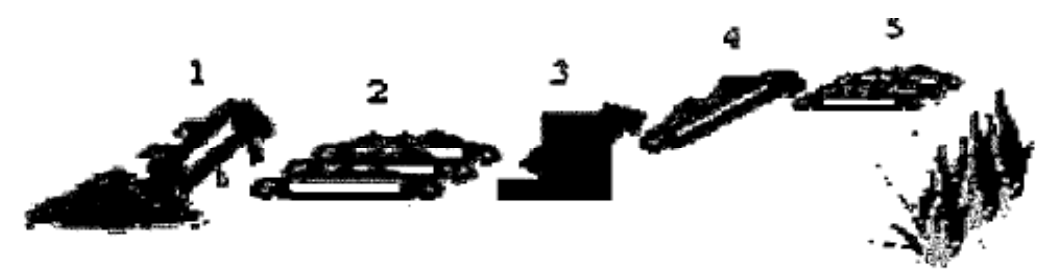

Figure 3. Electrical power station coal transportation systems [29]

The example is solved by use of the data given in Table 1. Each element of the system is considered as a init with total failures. Table 2 contains the data of the piecewise cumulative boiler demand curve.

\section{B. Execution and Firefly Algorithm Parameters}

The simulation results depend greatly on the firefly algorithm parameters values. We set $\alpha_{\max }=20, \alpha_{\min }=0.01, \beta_{0}{ }_{0}=0.5, \gamma=0.01$. To implement the algorithm presented in this paper, a $\mathrm{C}++$ code was written and executed on a machine with a AMD-A4-3300M APU 1.9GHz CPU and 4GB RAM. A range of values of the parameters was tested to choose the appropriate ones. Though a combination of finely tuned parameters' values leads to better convergence of metaheuristic algorithms, to choose the best combination is very difficult and becomes a drawback of these algorithms.

Table 1. Characteristics of the system elements available in the market [5]

\begin{tabular}{|c|c|c|c|c|c|}
\hline & Subsystems & Versions & $\begin{array}{c}\text { Reliability } \\
\text { A }\end{array}$ & $\begin{array}{c}\text { Cost } \\
C(\operatorname{mln} \$)\end{array}$ & $\begin{array}{c}\text { Capacity } \\
\Xi(\%)\end{array}$ \\
\hline \multirow{7}{*}{1} & \multirow{7}{*}{ Primary feeder } & 1 & 0.980 & 0.590 & 120 \\
\hline & & 2 & 0.977 & 0.535 & 100 \\
\hline & & 3 & 0.982 & 0.470 & 85 \\
\hline & & 4 & 0.978 & 0.420 & 85 \\
\hline & & 5 & 0.983 & 0.400 & 48 \\
\hline & & 6 & 0.920 & 0.180 & 31 \\
\hline & & 7 & 0.984 & 0.220 & 26 \\
\hline \multirow{5}{*}{2} & \multirow{5}{*}{ Primary conveyor } & 1 & 0.995 & 0.205 & 100 \\
\hline & & 2 & 0.996 & 0.189 & 92 \\
\hline & & 3 & 0.997 & 0.091 & 53 \\
\hline & & 4 & 0.997 & 0.056 & 28 \\
\hline & & 5 & 0.998 & 0.042 & 21 \\
\hline \multirow{4}{*}{3} & \multirow{4}{*}{ Stacker- reclaimer } & 1 & 0.971 & 7.525 & 100 \\
\hline & & 2 & 0.973 & 4.720 & 60 \\
\hline & & 3 & 0.971 & 3.590 & 40 \\
\hline & & 4 & 0.976 & 2.420 & 20 \\
\hline \multirow{9}{*}{4} & \multirow{9}{*}{ Secondary feeder } & 1 & 0.977 & 0.180 & 115 \\
\hline & & 2 & 0.978 & 0.160 & 100 \\
\hline & & 3 & 0.978 & 0.150 & 91 \\
\hline & & 4 & 0.983 & 0.121 & 72 \\
\hline & & 5 & 0.981 & 0.102 & 72 \\
\hline & & 6 & 0.971 & 0.096 & 72 \\
\hline & & 7 & 0.983 & 0.071 & 55 \\
\hline & & 8 & 0.982 & 0.049 & 25 \\
\hline & & 9 & 0.977 & 0.044 & 25 \\
\hline \multirow{4}{*}{5} & \multirow{4}{*}{ Secondary conveyor } & 1 & 0.984 & 0.986 & 128 \\
\hline & & 2 & 0.983 & 0.825 & 100 \\
\hline & & 3 & 0.987 & 0.490 & 60 \\
\hline & & 4 & 0.981 & 0.475 & 51 \\
\hline
\end{tabular}

Table 2. Parameters of the cumulative load demand curve [5]

\begin{tabular}{ccccc}
$\mathbf{W}_{\mathbf{m}} \mathbf{( \% )}$ & 100 & 80 & 50 & 20 \\
$\mathbf{T}_{\mathbf{m}}(\mathbf{h})$ & 4203 & 788 & 1228 & 2536 \\
\hline
\end{tabular}


C. Results and Discussion

Table 3. Solutions omparison for different values of $\mathrm{A}_{0}$

\begin{tabular}{|c|c|c|c|c|c|c|c|c|c|}
\hline $\mathbf{A}_{0}$ & Subsystem & GA [5] & ACO [8] & TS [9] & SP/TG [30] & HS [7] & IA [10] & $\mathrm{PSO} / \mathrm{LS}$ [6] & FF \\
\hline & & \multicolumn{8}{|c|}{ Optimal Solution } \\
\hline \multirow{5}{*}{0.970} & 1 & $2(2)$ & $2(2)$ & - & - & - & - & - & $2(2)$ \\
\hline & 2 & $5(6)$ & $3(3)$ & - & - & - & - & - & $3(3)$ \\
\hline & 3 & $1(1)$ & $1(1)$ & - & - & - & - & - & $1(1)$ \\
\hline & 4 & $9(6)$ & $8(3), 9(3)$ & - & - & - & - & - & $7(3)$ \\
\hline & 5 & $3(3)$ & $3(2), 4(1)$ & - & - & - & - & - & $3(3)$ \\
\hline Cost (mln \$) & & 10.581 & 10.602 & - & - & - & - & - & 10.55 \\
\hline A & & 0.970 & 0.970 & - & - & - & - & - & 0.970 \\
\hline \multirow{5}{*}{0.975} & 1 & $4(2), 6(1)$ & $4(2), 6(1)$ & $2(2)$ & $4(2), 6(1)$ & $4(2), 6(1)$ & $4(2), 6(1)$ & $1(1), 2(1)$ & $2(1), 7(1)$ \\
\hline & 2 & $5(6)$ & $5(6)$ & $3(2)$ & $5(6)$ & $5(6)$ & $5(6)$ & $6(1)$ & $1(1), 3(2)$ \\
\hline & 3 & 1(1),4(1) & 1(1),4(1) & 2(2) & $1(1), 4(1)$ & $1(1), 4(1)$ & 1(1),4(1) & $1(2)$ & $1(1), 4(1)$ \\
\hline & 4 & $7(3)$ & $7(3)$ & $7(3)$ & $7(3)$ & $7(3)$ & $7(3)$ & $3(1)$ & $6(1), 7(2)$ \\
\hline & 5 & $4(3)$ & $4(3)$ & 2(1) & $4(3)$ & $4(3)$ & $4(3)$ & $3(1)$ & $3(1), 4(2)$ \\
\hline Cost (mln \$) & & 12.855 & 12.855 & 16.450 & 12.855 & 12.855 & 12.855 & 12.855 & 12.7649 \\
\hline A & & 0.9761 & 0.9761 & 0.9774 & 0.976 & 0.976 & 0.976 & 0.976 & 0.9753 \\
\hline \multirow{5}{*}{0.980} & 1 & $4(2), 6(1)$ & $4(2), 6(1)$ & $2(2)$ & $4(2), 6(1)$ & $4(2), 6(1)$ & $4(2), 6(1)$ & $1(1), 2(1)$ & $2(1)$ \\
\hline & 2 & $3(2)$ & $3(2)$ & $5(6)$ & $3(2)$ & $2(1), 5(1)$ & $2(1), 5(1)$ & 2(1) & $2(2), 4(1)$ \\
\hline & 3 & 2(1),3(2) & $2(1), 3(2)$ & 2(3) & $2(1), 3(2)$ & $3(1), 2(2)$ & $3(1), 2(2)$ & $1(1), 2(1)$ & $2(1), 3(2)$ \\
\hline & 4 & $7(3)$ & $7(3)$ & $7(3)$ & $7(3)$ & $7(3)$ & $7(3)$ & 3(1) & $4(1), 7(2)$ \\
\hline & 5 & $3(2), 4(1)$ & $3(2), 4(1)$ & $2(1)$ & $3(2), 4(1)$ & $2(1), 4(1)$ & $2(1), 4(1)$ & $1(1), 2(1)$ & $3(1), 4(2)$ \\
\hline Cost (mln \$) & & 14.770 & 14.770 & 16.520 & 14.770 & 15.794 & 15.794 & 14.770 & 14.572 \\
\hline A & & 0.9820 & 0.9820 & 0.9808 & 0.9820 & 0.9826 & 0.9826 & 0.980 & 0.9817 \\
\hline \multirow{5}{*}{0.990} & 1 & $4(2), 6(1)$ & $4(2), 6(1)$ & $2(2)$ & $4(2), 6(1)$ & $4(2), 7(1)$ & $4(2), 7(1)$ & $1(1), 2(1)$ & $2(1)$ \\
\hline & 2 & $3(2)$ & $3(2)$ & $3(2)$ & $3(2)$ & $2(1), 5(1)$ & $2(1), 5(1)$ & $2(1)$ & $2(5), 4(3)$ \\
\hline & 3 & $2(2), 3(1)$ & $2(2), 3(1)$ & 2(3) & $2(2), 3(1)$ & $2(2), 3(1)$ & $2(2), 3(1)$ & $1(1), 2(1)$ & $1(3), 2(2)$ \\
\hline & 4 & $7(3)$ & $7(3)$ & $7(3)$ & $7(3)$ & $7(3)$ & $7(3)$ & $3(1)$ & 2(6), $9(3)$ \\
\hline & 5 & $4(3)$ & $4(3)$ & $4(3)$ & $4(3)$ & $4(3)$ & $4(3)$ & $3(1)$ & $4(3)$ \\
\hline Cost (mIn \$) & & 15.870 & 15.870 & 17.050 & 15.870 & 15.959 & 15.959 & 15.870 & 15.560 \\
\hline A & & 0.990 & 0.990 & 0.9937 & 0.9920 & 0.9931 & 0.9931 & 0.992 & 0.992 \\
\hline
\end{tabular}


The studied system consists of five subsystems connected in series, and for each subsystems, from 4 to 9 different elements types connected in parallel are available. The reliability index $\mathrm{A}_{0}$ has been set to four different values, namely $0.97,0.975,0.98$ and 0.99 , to create three variations of the problem. The objective is to select the optimal combination of elements used in series-parallel structure of the system. This has to correspond to the minimal total cost with regard to the selected level of the system availability. For a fair comparison, the same number of iterations (500), and the populations (150).

The comparisons between the best solution of firefly algorithm (FA) and the best solutions obtained by the methods in references $[5,6,7,8,9,10,30]$ are provided in Table 3 . Note that in these references the proposed algorithm is of a stochastic nature. The firefly algorithm on the redundancy optimization problem (ROP) for a multi-state system (MSS) with heterogeneous redundancy generally yields better solutions with a lower cost and reasonable reliability than the other algorithms. We take, for example, for reliability level $\left(\mathrm{A}_{0}=0.975\right)$ the solutions found by all algorithms are better than those by Tabu search [9], for another example the reliability level $\left(\mathrm{A}_{0}=0.98\right)$ the solutions found by all algorithms are better than those by HS [7], IA [10] and TS [9].

\section{Conclusion}

In conclusion, in this paper we have shown that the hybrid approach which combines between the UMGF and the FA system is an interesting approach to solve the Redundancy Optimization Problem with regard to heterogeneous multi-state series-parallel structures, allowing a maximum of two types of redundant components in each subsystem. The FA metaheuristic selects elements from a list of available products according to their reliability, nominal capacity (productivity) and unit cost. This approach allows us to minimize the total investments cost subject to availability constraint. The results are better compared to the methods in the literature in terms of solutions not computing time.

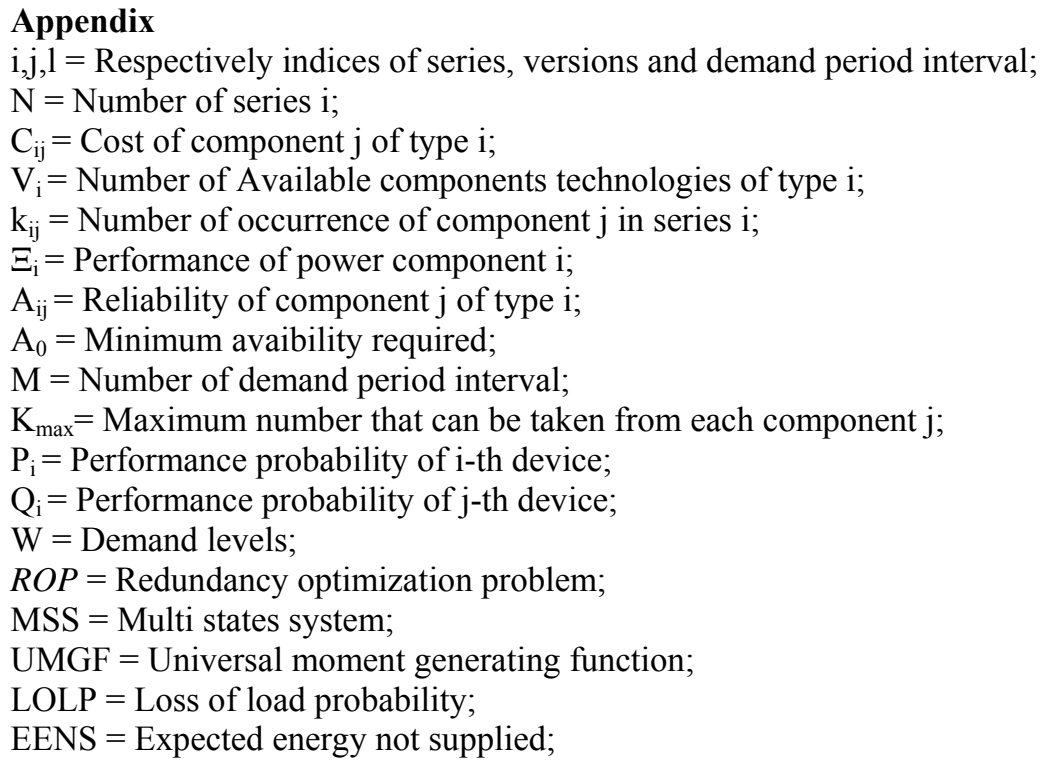

\section{References}

[1] F. A. Tillman, C. L. Hwang and W. Kuo, "Optimization, Techniques for System Reliability with Redundancy”, IEEE Transactions on Reliability, Vol. 26, No. 3, 1977.

[2] W. Kuo and V. R. Prasad, "An Annotated Overview of System Reliability Optimization", IEEE Transaction on Reliability, Vol. 49, No. 2, pp. 176-187, 2000. 
[3] I. Ushakov, "Optimal Standby Problems and a Universal Generating Function", Sov. J. Compt. Syst. Sci,Vol. 25, No. 4, pp. 79-82, 1987.

[4] G. Levitin, A. Lisniaski, H. Ben haim and D. Elmakis, "Power System Structure Optimization Subject to Reliability Constraints", Electric Power System Research, Vol. 39, pp. 145-152, 1996.

[5] G. Levitin, A. Lisnianski, H. Ben Haim and D. Elmakis, "Structure Optimization of Power System with Different Redundant Elements", Electric Power Systems Research, Vol. 43, No. 1, pp. 19-27. 1997.

[6] Y. Wang and L. Li, "Heterogeneous Redundancy Allocation for Series-Parallel MultiState Systems Using Hybrid Particle Swarm Optimization and Local Search", IEEE Transactions on Systems Man and Cybernetics Part, Vol. 42, No. 2; March 2012.

[7] A. Rami, "Design and Meta-heuristics Methods Optimization for Electro-Energetics System", PHD thesis, Université de Sidi Bel Abbes, Algeria, 2010.

[8] M. Agarwal, V.K. Sharma, "Ant Colony Optimization Algorithm for Heterogeneous Redundancy Allocation in Multi-State Series-Parallel Systems", International Journal of Reliability, Quality and Safety Engineering, Vol. 16, No. 2, pp.86-163, 2009.

[9] M. Ouzineb, M. Nourelfath and M. Gendreau, "Availability Optimization of SeriesParallel Multi-State Systems Using a Tabu Search Meta-heuristic", IEEE 2006.

[10] A. Rami, A. Zeblah, H. Hamdaoui, Y. Massim, F. Harrou, "An efficient Artificial Immune Algorithm for Power System Reliability Optimisation", International Journal of Power and Energy Conversion, Vol. 1, No. 2/3, pp. 178 - 197, 2009.

[11] J. Kennedy and R. C. Eberhart, "Particle swarm optimization", IEEE International Conference on Neural Networks, Piscataway, NJ, Seoul, Korea, Vol. 4, pp. 1942-1948, 1995.

[12] W. Coit and A. E. Smith, "Optimization Approaches to the Redundancy Allocation Problem for Series-Parallel Systems", Proceeding of the Fourth Industrial Engineering Research Conference, May 1995.

[13] M. Nourelfath, N. Nahas and A. Zeblah, "An Ant Colony Approach to Redundancy Optimization for Multi-State System", International Conference on Industrial Engineering and Production Management, Porto-Portugal, 2003.

[14] Y. Massim, A. Zeblah, A. Ghoraf and R. Meziane, "Reliability Evaluation of Multi-State Series-Parallel Power System under Multi-States Constraints", Electrical Engineering Journal, Springer Verlags, Vol. 87, pp. 327-336, 2005.

[15] R. Billiton, R. Allan, "Reliability of Power Systems", Pitman, London, 1984.

[16] M. Amara, R. Meziane and A. Zeblah, "Cost Optimization for Series-Parallel Petroleum Transportation Pape-Lines under Reliability Constraints", Journal of Engineering Research and Applications, Vol. 4, No. 1, pp. 302-310; January 2014.

[17] G. Levitin, A. Lisnianski, H. Ben Haim and D. Elmakis, "Redundancy Optimization for Series-Parallel Multi-State Systems", IEEE Transactions on Reliability, Vol. 47, No. 2, pp. 165-172, 1998.

[18] O. Bendjeghaba, D. Ohahdi and A. Zeblah, "Hybrid Approach for Redundancy Optimization of Multi-State Power System", Leonardo Journal of Sciences, PP. 115-130, 2007.

[19] K. Reinschke, "System of Elements with Many States", Radio i Svyaz, Moscow, 1985.

[20] E. El-Neweihi and F. Proschan, "Degradable Systems a Survey of Multistates System Theory", Communications in Statistics: Theory and Methods, Vol. 13, pp. 405-432, 1984.

[21] I. Ushakov, "Universal generating function", Journal Compt. Syst. Sci, Vol. 24, No. 5, pp. 118-129, 1986.

[22] Y. Liu, H. Z. Huang, Z. Wang, Y.F. LI, and X.L. ZHANG, "Joint Optimization of Redundancy and Maintenance Staff Allocation for Multi-State Series-Parallel Systems", Eksploatacja i Niezawodnosc - Maintenance and Reliability, Vol. 14, No. 4, pp. 312-318, 2012. 
[23] X. S. Yang, "Nature-Inspired Metaheuristic Algorithm", Luniver Press, Beckington, 2008.

[24] X. S. Yang, "Engineering Optimization an Introduction with Metaheuristic Applications" , New York, Wiley, 2010.

[25] L. Dekhici and K. Belkadi, "Metaheuristic Fireflies Discrete for the Flow Shop Hybrid", Cari 2012.

[26] K. Durkota, "Implementation of a Discrete Firefly Algorithm for the QAP Problem Within the Sage Framework", Bse Thesis, Czech Technical University, 2011.

[27] L. S. Coelho, D. L. A. Bernert and V. C. Mariani, "A chaotic firefly algorithm applied to reliability-redundancy optimization", Proceedings of IEEE, Congress on Evolutionary Computation, New Orleans, LA, USA, pp. 517-521, 2011.

[28] A. H. Gandomi, X. S. Yang and A. H. Alavi, "Mixed variable structural optimization using firefly algorithm", Computers and Structures, 89, 2325-2336, 2011.

[29] M. Ouzineb, "Efficient Heuristic for Serie-Parallel Performance Optimization”, PHD thesis, Université de Montréal, Canada, March 2009.

[30] M. Ouzineb, M. Nourelfath, M. Gendreau, "A Heuristic Method for Non-Homo- geneous Redundancy Optimization of Series-Parallel Multi-State Systems", Journal of Heuristics, Vol. 17, No. 1, pp. 1-22, 2011.

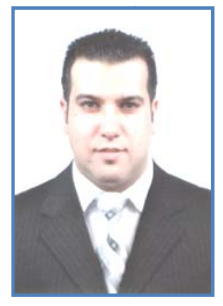

Khaled Guerraiche was born in Oran, Algeria, is a Phd student in the department of Electrical, Engineering faculty, university of sciences and technology of Oran, Mohamed Boudiaf (Algeria). He received his BS degree in electrical engineering from the Electrical Engineering Institute of The University of Sciences and Technology of Oran (USTO) in 2004, the MS degree from the Electrical Engineering Institute of The University of Sciences and Technology of Oran (USTO) in 2008. His research interests include operations, planning and economics of electric energy systems, as well as optimization theory and its applications.

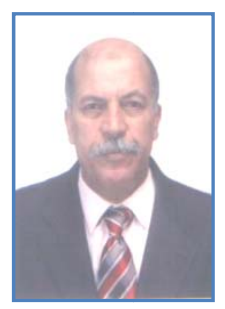

Mostefa Rahli was born in 1949 in Mocta-Douz, Mascara, Algeria. He received his BS degree in electrical engineering from the Electrical Engineering Institute of The University of Sciences and Technology of Oran (USTO) in 1979, the MS degree from the Electrical Engineering Institute of The University of Sciences and Technology of Oran (USTO) in 1985, and the $\mathrm{PhD}$ degree from the Electrical Engineering Institute of The University of Sciences and Technology of Oran (USTO) in 1996. From 1987 to 1991, he was a visiting professor at the University of Liege (Monteore's Electrical Institute) Liege (Belgium) where he worked on Power Systems Analysis analysis under Professors Pol Pirotte and Jean Louis Lilien. He is currently Professor of electrical engineering at The University of Sciences and Technology of Oran (USTO), Oran, Algeria. His research interests include operations, planning and economics of electric energy systems, as well as optimization theory and its applications. rahlim@yahoo.fr 


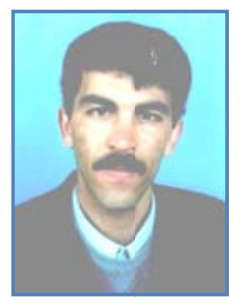

Abdelkader Zeblah was born in 1965 in Sfisef, Sidi Belabbes, Algeria. He received his $\mathrm{BS}$ degree in electrical engineering from the Electrical Engineering Institute of The University of Sidi Belabbes (Algeria) in 1990, the MS degree from the Electrical Engineering Institute of The University of Sciences and Technology of Oran (USTO) in 1993, and the PhD degree from the Electrical Engineering Institute of The University of Sciences and Technology of Oran (USTO) in 2001. He is currently Professor of electrical engineering at The University of Sidi Belabbes (Algeria). His research interests include operations, planning and economics of electric energy systems, as well as optimization theory and its applications.zeblaha@yahoo.fr

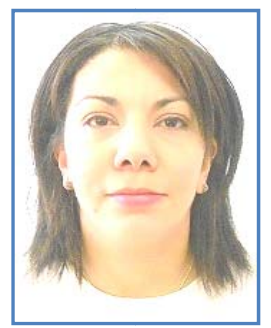

Latifa Dekhici is a Phd student in the department of computers sciences, sciences faculty, university of sciences and technology of Oran, Mohamed Boudiaf. In 2002, she had an engineering diploma in computer sciences in software engineering. She had his magister diploma in artificial intelligence and forms recognition in 2005. She works in both power dipatching optimization and the modeling, simulating and scheduling of the operating theatres of Oran. She had already published 5 journals papers and she has among 21 manuscripts in several conferences. L. Dekhici is attached to LAMOSI (Laboratory of Simulation of Systems of Industries).latifa.dekhici@univ-usto.dz 